\title{
NEW RECORDS OF THE TRIBE Cerambycini (Coleoptera: Cerambycidae: Cerambycinae) FROM VIETNAM
}

\author{
Cao Thi Quynh Nga*, Khuat Dang Long, Ta Huy Thinh \\ Institute of Ecology and Biological Resouces, VAST, *cqnga119@yahoo.com
}

\begin{abstract}
The paper listed 50 species of 18 genera belonging to the tribe Cerambycini from Vietnam, of the total 50 species there are five species newly recorded for the Vietnam's fauna, viz., Aeolesthes (Pseudaeolesthes) pericalles Gressitt \& Rondon, 1970 and one species of the genus Aeolesthes s.s. are Aeolesthes sinensis Gahan, 1890; Derolus ornatus Gressitt \& Rondon, 1970; Dymasius maculatus Gressitt \& Rondon, 1970; Massicus raddei (Blessig, 1872). Diagnosis of all genera are presented with notes on distribution. An illustrated key to 18 genera of the tribe Cerambycini is also provided.
\end{abstract}

Keywords: Coleoptera, Cerambycidae, Cerambycinae, Cerambycini, distribution, new record, Vietnam.

\section{INTRODUCTION}

The Cerambycini is one of the generically diverse tribes in the Cerambycinae. According to Gressitt \& Rondon (1970) [13], the subfamily Cerambycinae is divided into 21 tribes, there are 18 tribes recorded in Vietnam. The tribe Cerambycini is a large of longhorn beetles that occurs throughout the world, but is most diverse in the Neotropical, Oriental and Palearctic regions. The tribe Cerambycini comprises largesized species, i.e. from $10 \mathrm{~mm}$ to $74 \mathrm{~mm}$. Up to now, there were about 5000 known species of the tribe Cerambycini in the world. Of the total extant species from the Oriental region, 60 species occur in Laos (Gressitt \& Rondon, 1970) [13], 40 species in China (Hua, 2009) [34], 92 species in Borneo (Heffern, 2005) [15], 46 species in Thailand [61] and 12 species in Sri Lanka (Makihara et al., 2008) [37].

In Vietnam, the species of the tribe Cerambycini have been reported in papers scattered in the literature Vitalis Salvaza (1919); Mai Phu Qui et al (1981); Ta Huy Thinh \& Hoang Vu Tru (2005); Hoang Vu Tru, Cao Thi Quynh Nga \& Ta Huy Thinh (2009); Hoang Vu Tru, Ta Huy Thinh \& Cao Thi Quynh Nga (2011); Cao Thi Quynh Nga \& Khuat Dang Long (2014); Hayashi (1992) and Hua Li-zhong (2009).

Our paper continues to gain an understanding of the fauna of Vietnam and in this paper some of new records of the tribe
Cerambycini from Vietnam. Additionally, the illustrated key to the genera of this tribe is also provided.

\section{MATERIALS AND METHODS}

Studied specimens were from the insect collection of the Derpartment of Insect Systematics (DIS) at the Institute of Ecology and Biological Resource (Hanoi, Vietnam). All the specimens were collected by netting and light trapping at several localities from Vietnam. The specimens of the longhorn beetles were collected in several surveys organized by entomologists of Institute of Ecology and Biological Resources (IEBR).

Terminology used in this paper follows Gressitt \& Rondon (1970). Abbreviations of localities used in this paper are as follows: N: North; S: South; C: Central; NC: North Central; NE: Northeast; NW: Northwest; SW: Southwest; W: West; the old name of localities used in the literature: Annam=North Central; Cochin=Southern Vietnam and Tonkin= Northern Vietnam; NP=National Park. The asterisk indicates species newly recorded for the fauna of Vietnam. In-country distribution of the species is followed in order of areas, provinces (in brackets) from north to south, and outside Vietnam distribution of species follows in the alphabetical order.

\section{RESULTS AND DISCUSSION}




\section{Taxonomy}

Tribe Cerambycini Mulsant, 1839: Gahan, 1906: 119

Diagnosis: Antenae longer than body in male. Frons usually has furrow or grooved between eyes (figs 3a-f); eyes coarsely facetted. Procoxa cavity usually rounded or angulate on the outer side. Mid coxal cavity open to epimeron externally. Intercoxal process of prosternum wide, broadened distally.

In Vietnam, the tribe Cerambycini included
18 genera, such as Aeolesthes Gahan, 1890; Cyriopalus Pascoe, 1866; Derolus Gahan, 1891; Dialeges Pascoe, 1856; Diorthus Gahan, 1891; Dymasius Thomson, 1864; Falsoxeanodera Pic, 1923; Hoplocerambyx Thomson, 1864; Margites Gahan, 1891; Massicus (Pascoe, 1867); Nadezhdiella Plavilstshikov, 1931; Neocerambyx Thomson, 1860; Plocaederus Thomson, 1861; Rhytidodera White, 1853; Trachylophus Gahan, 1888; Trirachys Hope, 1841; Xoanodera Pascoe, 1857 and Zatrephus Pascoe, 1857.

\section{Key to the genera of Cerambycini from Vietnam}

(The following key is adapted from Gressitt and Rondon, 1970)

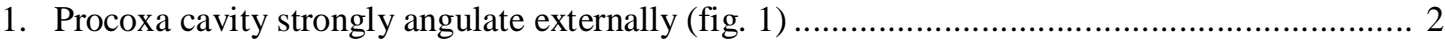

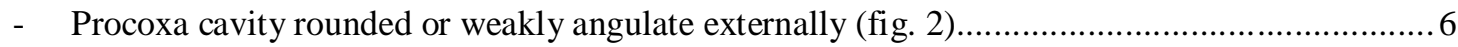

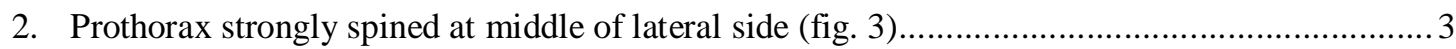

- Prothorax not spined at middle of lateral side, rarely with a blunt tubercle or

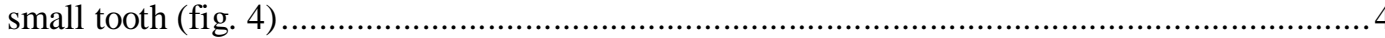

3. Head with a weakly carinate between upper eye lobe, scape segment arched externally (fig. 5). Elytra less than 3 times as long as broad Plocaederus

- Head with a sulcate between upper eye lobe, scape not arched externally (fig. 6). Elytron more than 3 times as long as broad Nadezhdiella

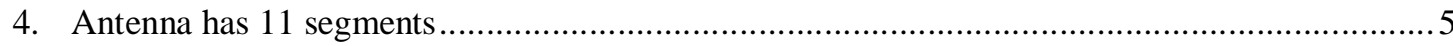

- Antenna has 12 segments, from 3-11 with an external process longer than

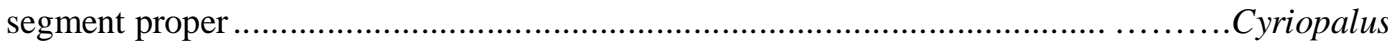

5. Antenna segment 3 not much longer than 4, 3 and 4 often swollen apically or preapically (fig. 7). Prothorax often obtuse or blunty toothed at lateral side ..............Neocerambyx

- Antenna segment 3 much longer than 4, 3 and 4 not swollen (fig. 8). Prothorax not toothed at side Massicus

6. Antenna segments $3-10$ or 5-10 spined or toothed endoapically …........................................ 7

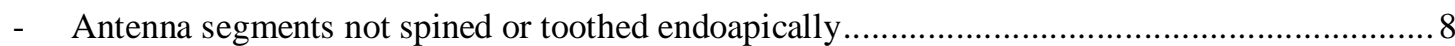

7. Head with a long deep groove between upper eye lobe (fig. 9). Prothorax not spined at middle of lateral side. Elytral pubescence vague Hoplocerambyx

- Head with a partial ridge between upper eye lobe (fig. 10). Prothorax strongly spined at middle of lateral side. Elytral pubescence varied presenting changing patterns

Trirachys

8. Head with a median carina between upper eye lobe (fig. 11). Antenna usually more than 2.5 times as long as body in male. Elytral pubescence producing highly changeable patterns according to incidence of light Aeolesthes

- Head without a median carina between eyes (fig. 12). Antenna not more than 2,5 times as long as body in male.

9. Prothorax longer than broad. Hind tarsal segment 1 longer than $2+3$ combined ...................... 10

- Prothorax not longer than broad. Hind tarsal segment 1 not longer than $2+3$ combined 
10. Antenna fringed beneath in male (fig. 13). Elytral pubescence producing highly changeable patterns according to incidence of light Dialeges

- Antenna not fringed beneath in male (fig. 14). Elytral pubescence usually uniform .....Dymasius

11. Antenna slender, longer than 1.4 times as long as body in male. Hind tarsal segment 1 as long as 2+3 combined (fig. 15)

- Antenna stout or expanded externally, less than 1.4 times as long as body in male. Hind tarsal segment 1 shorter than $2+3$ combined (fig. 16)

12. Prosternal intercoxal process gradually sloping posteriorly (fig. 17). Antenna not longer than $1,5 \mathrm{x}$ as long as body

- Prosternal intercoxal process truncate, subvertical or tuberculate posteriorly (fig. 18)............. 13

13. Femora with a carinate on each side of hind edge. Prosternal intercoxal process truncate or subvertical posteriorly. Antenna scape without a narrow cicatrix at apex (fig. 19) .......... Derolus

- Femora without a carinate on each side of hind edge. Prosternal intercoxal process more or less tuberculate posteriorly. Antenna scape with a narrow cicatrix at apex (fig. 20)..... Diorthus

14. Antenna slender, longer than than body in male (fig. 21) ........................................... 15

- Antenna fairly stout, shorter than than body in male (fig. 22)......................................... 16

15. Elytron gradually sloped at side, without a ridge parallel to margin ......................Trachylophus

- Elytron not overhanging posteriorly at side, without a ridge parallel to margin... Falsoxeanodera

16. Prosternal intercoxal process gradually sloping posteriorly (fig. 23) ...................... Rhytidodera

- Prosternal intercoxal process tuberculate posteriorly (fig. 24)........................................ 17

17. Elytron overhanging posteriorly, with a ridge parallel to margin. Antenna segments 3-5 not shorter and swollen (fig. 25)

Xoanodera

- Elytron hardly overhanging posteriorly, without a ridge parallel to margin. Antenna segments 3-5 shorter and swollen (fig. 26) Zatrephus

\section{Systematics}

\section{Genus Aeolesthes Gahan, 1890}

Aeolesthes Gahan, 1890. Ann. Mag. Nat. Hist. Ser 6, 6: 250.

Diagnosis: Head with a median carina between upper eye lobe and two foveae in frons. Antenna usually more than $2,5 \mathrm{x}$ as long as body in male, barely longer than body in female. Prothorax not spined at side. Elytral pubescence producing highly changeable patterns according to incidence of light.

1. Aeolesthes (Pseudaeolesthes) aureopilosa Gressitt \& Rondon, 1970

Aeolesthes (Pseudaeolesthes) aureopilosa Gressitt \& Rondon, 1970: 63

Hoang Vu Tru et al., 2011: 983.

Specimens examined: Lam Dong (Da Lat): 1ðึ, 29.iv.2003 (DIS).

Distribution: S. Vietnam (Gia Lai, Lam Dong); Laos.
2*. Aeolesthes (Pseudaeolesthes) pericalles Gressitt \& Rondon, 1970

Aeolesthes (Pseudaeolesthes) pericalles Gressitt \& Rondon, 1970: 61

Specimens examined: Vinh Phuc (Tam Dao NP): 1, 500 m, 1.v.2004; 1q, 500 m, 1217.vi.1999 (DIS).

Distribution: NE. Vietnam (Vinh Phuc); Laos.

3. Aeolesthes (Pseudaeolesthes) rufimembris Pic, 1923

Aeolesthes (Pseudaeolesthes) rufimembris Pic, 1923: 8.

Gressitt \& Rondon, 1970: 63.

Specimens examined: Vinh Phuc (Tam Dao NP): 1ठ, $500 \mathrm{~m}$, vi.2000 (DIS); 4, $900 \mathrm{~m}$, vi.2000 (DIS); Son La (Copia NR): 19, $500 \mathrm{~m}$, 6-8.vi.2007 (DIS).

Distribution: NE. Vietnam (Vinh Phuc, Son La); Laos. 
4. Aeolesthes holosericea (Fabricius, 1787)

Cerambyx holosericea Fabricius, 1787: Gahan, 1906: 127.

Ta Huy Thinh \& Hoang Vu Tru, 2005: 303.

Synonym: Aeolesthes holocericea: Gahan, 1906.

Specimens examined: Thua Thien-Hue
(A Roang, A Luoi): 7ㅇ, 700 m, 3.v.2005 (DIS);


28.v.2006 (DIS); 9, 8ภ, $700 \mathrm{~m}, 29 . \mathrm{v} .2006$ (DIS); 2 , , >1,000 m, 5-8.v.2003.v.2006 (DIS).

Distribution: C. Vietnam (Thua Thien-Hue); India; Indonexia; Laos; Malaysia; S. China; Sri Lanka; Thailand.
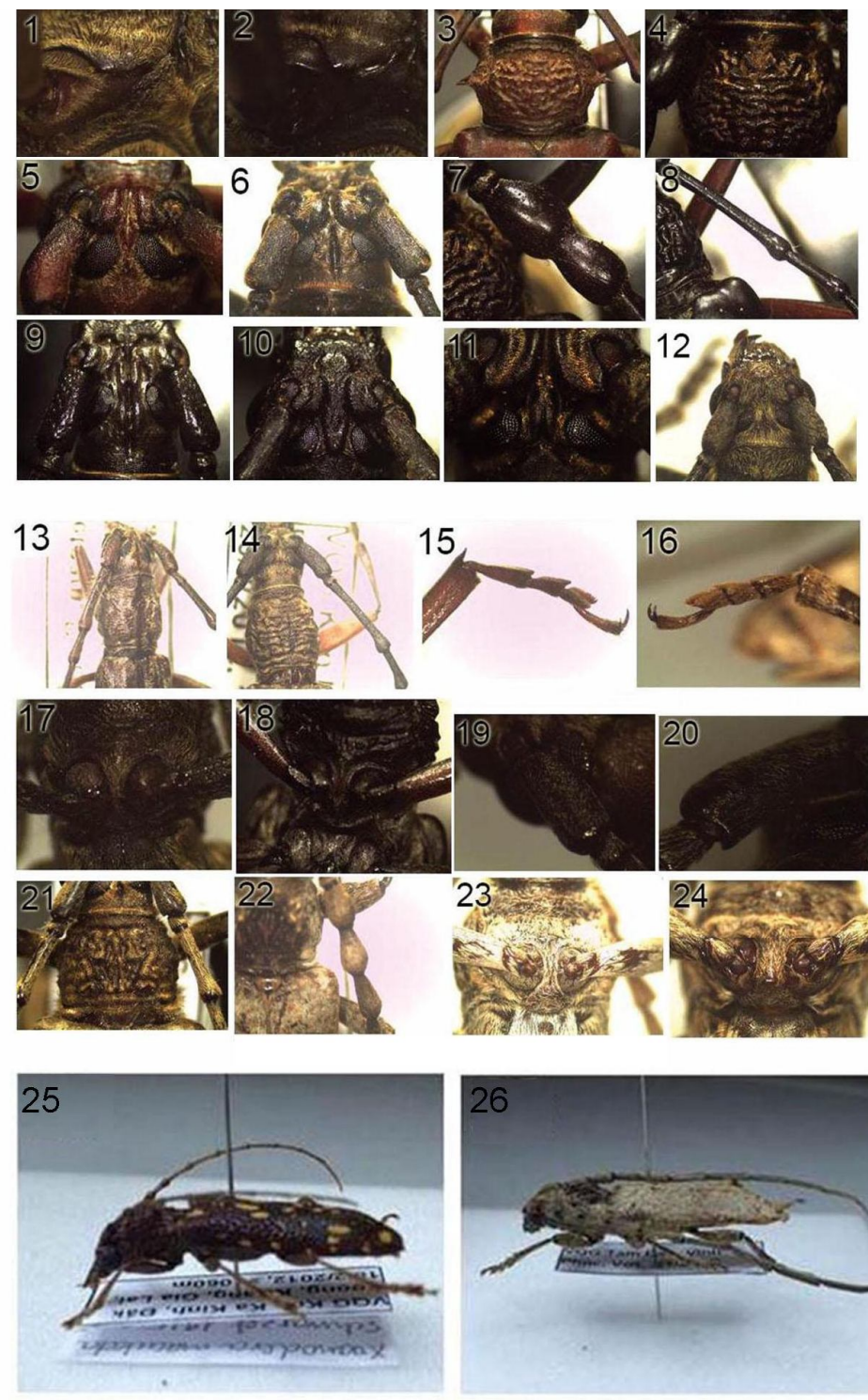

Figs 1-26. Cerambycini

1 (Procoxal cavity). Cyriopalus wallacei Pascoe; 2 (Procoxal cavity), 10 (Head). Trirachys orientalis Hope; 3 (Pronotum), 5 (Head). Plocaederus obesus Gahan; 4 (Head), 7 (Antenna). Neocerambyx grandis Gahan; 6 (Head). Nadezhdiella cantori (Hope); 8 (Antenna). Massicus suffusus Gressitt \& Rondon; 9 (Head). Hoplocerambyx spinicornis (Newman); 11 (Head). Aeolesthes induta (Newman); 12 (Head), 17 (Prosternal intercoxal process). Margites luteopubens Pic; 13 (Antenna). Dialeges undulatus Gahan; 14 (Antenna). Dymasius maculatus Gressitt \& Rondon; 15 (Hind tarsal), 18 (Prosternal intercoxal process), 19 (Cicatrix). Derolus volvulus (Fabricius); 16 (Hind tarsal), 22 (Antenna), 26 (Habitus). Zatrephus longicornis Pic; 20 (Cicatrix). Diorthus cinereus (Fabricius); 21 (Antenna). Trachylophus acutulus Holzschuh; 23 (Prosternal intercoxal process). Rhytidodera grandis White; 24 (Prosternal intercoxal process), 25 (Habitus). Xoanodera maculata Schwarzer.
5. Aeolesthes aurifaber (White, 1853)

Hammaticherus aurifaber White, 1853: 128.

Hoang Vu Tru et al., 2011: 983.
Specimens examined: Kon Tum (Chu Mom Ray NP): 1ð, 4.ii.2014 (DIS); Kien Giang (Phu Quoc NP): 1ठ, 11.x.2003 (DIS). 
Distribution: Vietnam (Kon Tum, Gia Lai, Kien Giang); Borneo island; Malaysia; Laos.

6. Aeolesthes induta (Newman, 1842)

Hammaticherus indutus Newman, 1842: 245.

Synonym: Aeolesthes induta: Gahan, 1906.

Specimens examined: Cao Bang (Phia Oac NR): 5q, 20̂, 29.iv-6.v.2012, net (DIS); Bac Kan (Ba Be NP): 19, 29.iv-1.v.2014, Pham Hong Thai coll. (DIS); Hoa Binh (Thuong Tien NR, Kim Boi): 1q, 27.iv.2012, net (DIS); Vinh Phuc (Tam Dao NP): 1, 900 m, 4.ii.2005; 4우, 5-11.v.2011, net (DIS); ThuaThien-Hue: 1, A Roang, A Luoi, 29.v.2006; 19, Tri Sao, 600 m, 7.v.2003, light trap (DIS); Quang Nam (Cha Val, Nam Giang): 1ㅇ, 29.iv.2005, light trap (DIS); Gia Lai (Kon Ka Kinh NP): 2q, 2ภ, 1230m, 24.ii.2012 (DIS).

Distribution: Vietnam (Cao Bang, Bac Kan, Hoa Binh, Vinh Phuc, Thua Thien-Hue, Quang Nam, Gia Lai); Laos; Myanmar; Philippines; S. China; Sri Lanka; Thailand.

7*. Aeolesthes sinensis Gahan, 1890

Aeolesthes sinensis Gahan, 1890: 255.

Specimens examined: Thua Thien-Hue (A Roang, A Luoi): 21우 15^, 700-760m, 35.v.2004, 28-29.v.2006 (DIS).

Distribution: C. Vietnam (Thua Thien-Hue); China (Taiwan); India; Laos; Myanmar.

Genus Cyriopalus Pascoe, 1866

Cyriopalus Pascoe, 1866. Proc. Zool. Soc. Lond, 1866: 530.

Diagnosis: Eyes coarse. Procoxa cavity strongly angulate externally. Antenna 12 segments. Prothorax strongly spined at middle of side. Elytral pubescence fairly uniform and bright red.

8. Cyriopalus wallacei Pascoe, 1866

Cyriopalus wallacei Pascoe, 1866: Gressitt \& Rondon, 1970: 59.

Ta Huy Thinh \& Hoang Vu Tru, 2005: 303. 1869.

Synonym: Cyriopalus wallacei: Wallacei,

Specimens examined: Thua Thien-Hue: 1 , A Roang, A Luoi, 700 m, 4.v.2004; 1으, Bach
Ma NP, 1,300 m, 6.xi.2002 (DIS); Dong Nai (Phu Ly, Vinh Cuu): 1우 8.ii.2008 (DIS).

Distribution: Vietnam (Thua Thien-Hue, Dong Nai); Laos.

\section{Genus Derolus Gahan, 1891}

Derolus Gahan, 1891. Ann. Mag. Nat. Hist, ser 6, 7: 26.

Diagnosis: Eyes divided. Antenna less than twice as long as the body in male, segment 1 without cicatrix at the apex. Procoxa cavity weakly or not at all angulate externally. Prothorax transverse, rounded at the middle of side. Prosternal intercoxal process subvertical. Elytra truncate or sometimes rounded at the apex. Femora with a fine carinate on each side of hind edge.

9. Derolus blaisei Pic, 1923

Derolus blaisei Pic, 1923: 8.

Gressitt \& Rondon, 1970: 74.

Specimens examined: Quang Binh (Truong Son, Quang Ninh): 1ð, 550 m, 10.v.2007 (DIS).

Distribution: Vietnam: (Quang Ninh, Quang Binh, Gia Lai); Laos.

10. Derolus griseonotatus Pic, 1923

Derolus griseonotatus Pic, 1923: 8.

Gressitt \& Rondon, 1970: 74.

Specimens examined: None.

Distribution: N. Vietnam; Laos.

11*. Derolus ornatus Gressitt \& Rondon, 1970

Derolus ornatus Gressitt \& Rondon, 1970: 76

Specimens examined: Quang Binh (Truong Son, Quang Ninh): 1ठ, 550 m, 10.v.2007 (DIS); Bac Kan (Ba Be NP): 1ð, 29.iv-1.v.2014 (DIS).

Distribution: NE. Vietnam (Bac Kan, Quang Ninh); Laos.

12. Derolus volvulus (Fabricius, 1801)

Cerambyx volvulus Fabricius, 1801: Gressitt \& Rondon, 1970: 74.

Hua Li-zhong, 2002: 204.

Specimens examined: Bac Kan (Ba Be NP): 1§, 19.v.2014 (DIS).

Distribution: NE. Vietnam (Bac Kan); 
China; India; Laos; Philippines.

\section{Genus Dialeges Pascoe, 1856}

Dialeges Pascoe, 1856. Trans. Ent. Soc. Lond. ser 2, 4: 46

Diagnosis: Eyes divided. Antenna fringed beneath in male, segment 3 longest and incurvate. Procoxa cavity weakly angulate externally. Prothorax slightly longer than broad, constricted at apex and base. Elytral pubescence producing highly changeable patterns according to incidence of light.

\section{Dialeges pauper Pascoe, 1856}

Dialeges pauper Pascoe, 1856: 47.

Gressitt \& Rondon, 1970: 85.

Specimens examined: Thua Thien-Hue (A Roang, A Luoi): 20, 19, 700m, 21.vii.2004, 3.v.2005, 28.v.2006 (DIS); Gia Lai (Chu Se): 3ㅇ, 20.vii.2012 (DIS); Dong Nai (Phu Ly, Vinh Cuu,): 1ㅇ, 2.i.2008; 1ठ, 4.viii.2008 (DIS).

Distribution: Vietnam (Thua Thien-Hue, Kon Tum, Gia Lai, Dong Nai); Assam; Bangladesh; Borneo; India; Laos; Malayxia; Myanmar; E. Pakistan; Thailand.

14. Dialeges undulatus Gahan, 1891

Dialeges undulatus Gahan, 1891: 23.

Hoang Vu Tru et al, 2011: 984.

Specimens examined: Cao Bang (Phia Oac NR): 1ㅇ, 22.V.2013 (DIS); Bac Kan (Ba Be NP): 29, 29.iv-1.v.2014, 19.v.2014 (DIS); Quang Tri : 10ิ, Pê Ke, 5.v.2005; 10, Khe Sanh, Huong Hoa, 400 m, 3.v.2006; 1ठ, Sa Mu, Huong Phung, Huong Hoa, 900-1,000 m, 4.vi.2006 (DIS); Quang Nam (Dong Giang): 10ิ, 27.v.2006 (DIS).

Distribution: Vietnam (Cao Bang, Bac Kan, Quang Tri, Quang Nam, Kon Tum, Gia Lai); China; Laos; Myanmar; Srilanka; Thailand.

\section{Genus Diorthus Gahan, 1891}

Diorthus Gahan, 1891. Ann. Mag. Nat. Hist, ser6, 7: 27.

Diagnosis: Antenna nearly twice as long as the body in the male, segment scape with a distinct narrow cicatrix at apex. Prosternal intercoxal process subtruncate posteriorly.
Elytra truncate at the apex. Femora finely carinate along each side to the ventral border.

15. Diorthus cinereus (Fabricius, 1793)

Cerambyx cinereus Fabricius, 1793: Gressitt \& Rondon, 1970: 71.

Tru et al, 2011: 984. 1953.

Synonym: Diorthus cinereus Lepesme,

Taurotagus brevipennis Mateu, 1963.

Diorthus (Diorthus) cinereus Gressitt \& Rondon, 1970.

Specimens examined: Quang Nam (Thach My, Nam Giang): 1ð̊, 29.v.2009 (DIS).

Distribution: Vietnam (Quang Nam, Kon Tum, Gia Lai); Aden; W. Africa; Baluchistan; India; Laos; Mauritius; N. Myanmar; Sri Lanka; Pakistan; Thailand.

\section{Genus Dymasius Thomson, 1864}

Dymasius Thomson, 1864. Syst. Ceramb., 234.

Diagnosis: Antenna not fringed beneathand much longer than body in the male. Prothorax longer than broad, slightly rounded in the middle on each side. Elytral pubescence usually uniform, truncate at the apex. Prosternal intercoxal process usually subvertical and somewhat tuberculate posterioly. Hind tarsal segment 1 sometimes longer than $2+3$ combine.

16. Dymasius cos Holzschuh, 1998

Dymasius cos Holzschuh, 1998: 65.

Holzschuh, 1998: 65.

Specimens examined: None.

Distribution: C. Vietnam (Thua Thien-Hue), S. Vietnam.

17. Dymasius (Microdymasius) gracilicornis (Gressitt, 1951)

Zegriades gracilicornis Gressitt, 1951: 147-148.

Holzschuh, 2010: 137-225.

Specimens examined: Vinh Phuc (Tam Dao NP): 1ô, 500 m, 25-28.vii.2011 (DIS).

Distribution: NE. Vietnam (Vinh Phuc); China; Laos.

18. Dymasius (Elydnus) simplex Gressitt \& Rondon, 1970 
New records of the tribe Cerambycini

Dymasius (Elydnus) simplex Gressitt \& Rondon, 1970: 81.

Specimens examined: Thua Thien-Hue (A Roang, A Luoi): 29, 700, 28.v.2006 (DIS).

Distribution: C. Vietnam ( Thua ThienHue); Laos.

19. Dymasius (Elydnus) vitreus Pascoe, 1885

Dymasius (Elydnus) vitreus Pascoe, 1885: 53.

Specimens examined: Thua Thien-Hue (A Roang, A Luoi): 1웅 700m, 29.v.2006 (DIS).

Distribution: C. Vietnam (Thua Thien-Hue); NE. Borneo; Laos.

20. Dymasius (s.s.) aureofulvescens Gressitt \& Rondon, 1970

Dymasius (s.str.) aureofulvescens Gressitt \& Rondon, 1970: 80.

Specimens examined: Cao Bang (Phia Oac NR): 1§, 25.v-27.vi.2012, Pham Hong Thai coll. (DIS); Bac Kan (Ba Be NP): 5우워, 29.iv-19.v.2014, Pham Hong Thai coll. (DIS); Quang Binh (Truong Son, Quang Ninh): $1 \delta^{\pi}$, 550m, 9.v.2007 (DIS).

Distribution: Vietnam (Cao Bang, Bac Kan, Quang Binh); Laos.

21*. Dymasius (s.s.) maculatus Gressitt \& Rondon, 1970

Dymasius (s.str.) maculatus Gressitt \& Rondon, 1970: 80 .

Specimens examined: Thua Thien-Hue (A Roang, A Luoi): 2ふ, 700 m, 4.v.2005, 28.v.2006 (DIS); Gia Lai (Kon Ka Kinh NP): 3ㅇ, 4ð̂, 1,230 m, 23.ii.2012 (DIS).

Distribution: Vietnam (Thua Thien-Hue, Gia Lai); Laos.

22. Dymasius verticosus Holzschuh, 2010

Dymasius verticosus Holzschuh, 2010: 137-225.

Holzschuh, 2010: 137-225.

Specimens examined: None.

Distribution: N. Vietnam; Laos.

Genus Falsoxeanodera Pic, 1923

Falsoxeanodera Pic, 1923. Exchange 2: 8.

Diagnosis: Antenna slender, longer than than body in male. Prothorax broader than long.
Procoxa cavity weak angulate externally. Hind tarsal 1 slightly shorter than $2+3$ combine. Elytron not overhanging posteriorly at side, without a ridge parallel to margin.

23. Falsoxeanodera maculata Pic, 1923

Falsoxeanodera maculata Pic, 1923: Hua Lizhong, 2002: 209.

Hua Li-zhong, 2002: 209.

Materials examined: None.

Distribution: Vietnam; China.

Genus Hoplocerambyx Thomson, 1864

Hoplocerambyx Thomson, 1864. Syst. Ceramb., 229.

Diagnosis: Head strongly exserted, with a long deep groove between eyes. Eyes deeply emarginate. Antenna segments 3-10 spined endoapically. Procoxa cavity weakly angulate externally. Prothorax narrower at the apex, transversely rugose with a smooth median behind middle. Elytral pubescence vague and truncate. Prosternal intercoxalprocess truncate posteriorly.

24. Hoplocerambyx spinicornis (Newman, 1842)

Hammaticherus spinicornis Newman, 1842: Gahan, 1906: 131.

Gressitt \& Rondon, 1970: 70.

Specimens examined: Vinh Phuc (Tay Thien, Tam Dao NP): 2ᄋ, $1 \hat{\jmath}, 500 \mathrm{~m}$, 18.v.2004; 6우 $550 \mathrm{~m}$, 5-11.v.2011, 1624.vi.2011, 5.v.2013 (DIS); Ninh Binh (Cuc Phuong NP): 5q, 3ふै, 1.vi.2003, 30.iv.2004, 3.v.2005, 24-26.v.2005, 1.v.2006 (DIS); Thanh Hoa (Lung Cao, Ba Thuoc): 1q, 500m, 1113.iv. 2002 (DIS); Nghe An (Cao Veu, Pu Mat): 2ㅇ, 20̂, 30.ix.2008 (DIS); Ha Tinh (Son Kim, Huong Son): 1q, 3.v.2004 (DIS); Quang Binh (Truong Son, Quang Ninh): 2ᄋ, $550 \mathrm{~m}$, 9.v.2007 (DIS); Quang Tri (Pe Ke, Dak Rong): 19 , 5.v.2005 (DIS); Thua Thien-Hue: 8 , , Bach Ma NP, 5-8.v.2003, 16-18.v.2012; 11 , 11 $\widehat{\jmath}, \mathrm{A}$ Roang, A Luoi, 700 m, 20-23.vii.2004; 4, 1ð̄, A Roang, A Luoi, 700 m, 3.v.2005; 20ㅇ, 12ð,, A Roang, A Luoi, 700 m, 28-29.v.2006; 5ㅇ, A Roang, A Luoi, 760 m, 4.v.2005 (DIS); Quang Nam: 3, 2今, Cha Val, Nam Giang, 27- 
29.iv.2005; 1ठ, Song Thanh NR, 30.iv.2005; 13우 1 $\hat{\jmath}$, Lo Xo, Phuoc My, Phuoc Son,


Son, 700-720 m, 24-26.v.2006, 17.vii.2009 (DIS); Gia Lai: 1 ${ }^{\lambda}$, Kon Ka Kinh NP, 1,230 m, 23.ii.2012; 1ð̄, Chu Se, KBang, 20.vii.2012 (DIS); Kon Tum: 1ð̄, Sa Son, Sa Thay, 24.v.2009 (DIS); Dak Lak (Chu Yang Sin NP): 29, 18.x.2012 (DIS); Ninh Thuan (Phuoc Binh NP): 3ㅇ, 300 m, 13-14.xi.2008 (DIS); Dong

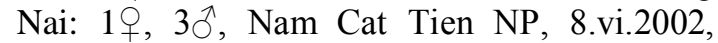
5.vii.2002, 7-15.vii.2012; 27ㅇ, 4^, Vinh Cuu NR, 25.vii-4.viii.2008 (DIS); Kien Giang (Phu Quoc NP): 2ㅇ, 19.ix.2012 (DIS).

Distribution: Vietnam (Vinh Phuc, Ninh Binh, Thanh Hoa, Nghe An, Ha Tinh, Quang Binh, Quang Tri, Thua Thien-Hue, Quang Nam, Gia Lai, Kon Tum, Dak Lak, Ninh Thuan, Dong Nai, Kien Giang); Afghanistan; India; Laos; Malayxia; Myanmar; Pakistan.

\section{Genus Margites Gahan, 1891}

Margites Gahan, 1891. Ann. Mag. Nat. Hist, ser 6, 7: 26.

Diagnosis: Head rather short. Antenna fairly slender, longer than the body in the male and shorter than the body in the female, not distinctly flattened on outer side.Prothorax rounded or obtusely subangulate at the middle of side. Elytra rounded or subtruncate at the apex. Prosternal intercoxal process gradually sloped. Hind tarsal segment 1 often as long as $2+3$ combine.

25. Margites aggregatus Holzschuh, 1999

Margites aggregatus Holzschuh, 1999: 64.

Holzschuh, 1999: 64.

Specimens examined: None.

Distribution: S. Vietnam.

26. Margites grisescens Pic, 1937

Margites grisescens Pic, 1937: Gressitt \& Rondon, 1970: 78

Gressitt \& Rondon, 1970: 78.

Specimens examined: None.

Distribution: NC. Vietnam; Laos.

27. Margites luteopubens Pic, 1926
Margites luteopubens Pic, 1926: 23

Specimens examined: Cao Bang (Phia Oac NR): 39, 29.iv-6.v.2012, 19.v.2013 (DIS); Thua Thien-Hue (A Roang, A Luoi): 19, 4.v.2005 (DIS); Gia Lai (Kon Ka Kinh NP): 1ㅇ, 1,230 m, 22.iii.2012 (DIS).

Distribution: Vietnam (Cao Bang, Thua Thien-Hue, Gia Lai); SW. China; Laos.

\section{Genus Massicus Pacsoe, 1867}

Massicus Pascoe, 1867. Ann. Mag. Nat. Hist, ser 3, 19: 319.

Diagnosis: Head with a short groove between eyes. Antenna a little longer than the body in the female and very longer in the male; antenna segment 3 much longer than 4, segment 3 and 4 not swollen. Prothorax not toothed at side. Elytral pubescence usually fairly uniform or bright red, truncated at apex.

28*. Massicus raddei (Blessig, 1872)

Masicus raddei (Blessig, 1872): Gressitt \& Rondon, 1970: 59.

Specimens examined: Lao Cai (Hoang Lien NP): 3q, 2200m, 30.iii.2005, 27-28.ix.2013 (DIS); Cao Bang (Phia Oac NR): 2q, 910.ix.2013 (DIS).

Distribution: Vietnam (Lao Cai, Cao Bang); N. China; Korean; Japan.

29.Massicus sufusus Gressitt \& Rondon, 1970

Massicus sufusus Gressitt \& Rondon, 1970: 59.

Specimens examined: Vinh Phuc (Tam Dao NP): 1 + $, 550 \mathrm{~m}, 22 . v i .2008$ (DIS); Ha Tinh: $1{ }^{\lambda}$, 300m, 24-30.v.2004; 2 ㅇ, Son Kim, Huong Son, 8.v.2004, Truong Xuan Lam coll. (DIS); Quang Binh (Truong Son, Quang Ninh): 2q, 550m, 9.v.2007; Quang Tri: 1+, Dak Rong, 625m, 5.v.2005; Quang Nam (Cha Val, Nam Giang): 1ठ, 27.iv.2005 (DIS).

Distribution: Vietnam (Vinh Phuc, Ha Tinh, Quang Binh, Quang Tri, Quang Nam); Laos.

30. Massicus trilineatus (Pic, 1933)

Dymasius trilineatus Pic, 1933: 12.

Gressitt \& Rondon, 1970: 59.

Specimens examined: Thua Thien-Hue (Bach Ma NP): 19, 1,200 m, 22.iv.2009 (DIS); 
New records of the tribe Cerambycini

Quang Nam (Deo Lo Xo, Quang Son): 1ð̂, 2.v.2005 (DIS); Gia Lai (Kon Ka Kinh NP): 1̊̄, 1,230 m, 15.iii.2012 (DIS) ; Kon Tum (Chu Mom Ray NP): 2, 31.iii-2.iv.2014 (DIS).

Distribution: Vietnam (Tonkin, Quang Nam, Gia Lai); China (Taiwan); Laos.

\section{Genus Nadezhdiella Plavilstshikov, 1931}

Nadezhdiella Plavilstshikov, 1931.

Diagnosis: Head with a sulcate between eyes; segment scape not arched externally. Procoxa cavity strongly angulate externally. Elytra has length less 3 times than broad.

31. Nadezhdiella cantori (Hope, 1845)

Cerambyx cantori Hope, 1845: Hua Li-zhong, 2009: 311.

Specimens examined: Son La (Muong Do, Phu Yen): 1ㅇ, 1ठ, 500-700 m, 6.8.iv.2008, Nguyen Quoc Binh coll.; 4엉 Moc Chau, 21.v.1996 (DIS); Cao Bang (Phia Oac NR): 2ㅇ, 10ิ, 25.v-7.vi.2012, 25.v.2014, Pham Hong Thai coll.; Vinh Phuc (Tam Dao NP): 29, 1.v.2000; 4⿳े, 1.vi.2000; 2へ, 25.v.2004 (DIS); Ha Tinh (Son Kim, Huong Son): 1ð, 3.v.2004 (DIS); Gia Lai (Kon Ka Kinh NP): 19, 1,230 m, 24.ii.2012 (DIS).

Distribution: Vietnam (Son La, Cao Bang, Vinh Phuc, Ha Tinh, Gia Lai); China.

32. Nadezhdiella fulvopubens (Pic, 1933)

Plocederus fulvopubens Pic, 1933: 27.

Holzschuh, 2005: 4. 1937

Synonym: Nadezhdiellaaurens Gressitt,

Nadezhdiella conica Chiang, 1942.

Materials examined: None.

Distribution: NE. Vietnam (Vinh Phuc); China; Laos; Thailand.

\section{Genus Neocerambyx Thomson, 1860}

Neocerambyx Thomson, 1860. Classif. Ceramb., 194

Diagnosis: Head deeply grooved between eyes. Antenna longer than body in male, shorter in female, segment 3 not much longer than 4 , segment 3 and 4 often swollen apically or preapically. Prothorax transverse, obtuse at side.
Procoxa cavity angulate externally. Elytra convex, pubescence usually fairly uniform, rounded at apex.

33. Neocerambyx vitalisi Pic, 1923

Neocerambyx vitalisi Pic, 1923: 12

Gressitt \& Rondon, 1970: 58

Specimens examined: Quang Tri (A Ngo, Dak Rong): 2ð, 200 m, 6.v.2005 (DIS).

Distribution: Vietnam (Quang Tri, S. Vietnam); Borneo; Indo-China; Laos.

34. Neocerambyx grandis Gahan, 1891

Neocerambyx grandis Gahan, 1891: 20

Ta Huy Thinh \& Hoang Vu Tru, 2005: 302304.

Specimens examined: Cao Bang (Phia Oac NP): 1ð̂, 25.v.2005 (DIS); Vinh Phuc (Tam Dao NP): 20, 1.vi.2002, 5-11.v.2011 (DIS); Quang Tri: 1ô, Huc Nghi, Dak Rong, 6.v.2005 (DIS); Kon Tum (Chu Mom Ray NP): 6우, 31.iii-2.iv.2014 (DIS).

Distribution: Vietnam (Cao Bang, Vinh Phuc, Quang Tri, Kon Tum); Laos; India.

35. Neocerambyx paris (Wood, 1821)

Cerambyx paris Wood, 1821: Gressitt \& Rondon, 1970: 58.

Ta Huy Thinh \& Hoang Vu Tru, 2005: 303

Specimens examined: Cao Bang (Phia Oac NP): 1ㅇ, 1ठ, 10.viii.2012, 9-10.ix.2013 (DIS); Quang Tri (Huc Nghi, Dak Rong): 1우 6.v.2005 (DIS).

Distribution: Vietnam (Cao Bang, Quang Tri); Laos; Myanmar; Thailand.

36. Neocerambyx tamdaoensis Hayashi, 1992

Neocerambyx taiwanensis Hayashi, 1992: 139.

Specimens examined: Thua Thien-Hue (A Roang, A Luoi): 2ㅇ, 29.v.2006, (DIS); Kon Tum (Chu Mom Ray NP): 1ㅇ, 1.iv.2014 (DIS).

Distribution: Vietnam (Vinh Phuc, Thua Thien-Hue, Kon Tum); China.

\section{Genus Plocaederus Thomson, 1861}

Plocaederus Thomson, 1861. Classif. Ceramb., 197 
Cao Thi Quynh Nga, Khuat Dang Long, Ta Huy Thinh

Diagnosis: Head oblique. Antenna longer than body in male, as long as body in female, 34 antennal segments thickened at apex and weakly angulate ectoapically, segment 5-9 strongly angulate ectoapically. Prothorax rugose, armed with sharp tooth. Procoxa transverse, cavity closed posteriorly; prosternum intercoxal process acute or vertical posteriorly. Elytra truncate at apex.

\section{Plocaederus obesus Gahan, 1890}

Plocaederus obesus Gahan, 1890: 51.

Ta Huy Thinh \& Hoang Tru, 2005: 303.

Specimens examined: Ha Tinh (Son Kim, Huong Son): 1ठ̉, 3.v.2004, Truong Xuan Lam coll. (DIS); Quang Tri (A Ngo, Dak Rong): $2 \hat{\jmath}$, 31.v.2006 (DIS); Gia Lai (Chu Se town): 2 , 1.v.2011 (DIS); Dak Nong (Dak Song): 1, 26.iv.2011 (DIS); Lam Dong: 1ठ, Dambri, Bao Loc, 600-800 m, 5.i.2003 (DIS); Can Tho: 2ð, Dai hoc Can Tho, ii-iv.1977 (DIS); Kien Giang: 1ㅇ, 1ठ, Hon Chong, <100 m, 17.vi.2005 (DIS).

Distribution: Vietnam (Ha Tinh, Quang Tri, Dak Lak, Dak Nong, Gia Lai, Lam Dong, Can Tho, Kien Giang); Andama island; Assam; S. China; India; Laos; Myanmar; Thailand.

38. Plocaederus ruficornis (Newman, 1842)

Cerambyx ruficornis Newman, 1842: 245.

Hoang Vu Tru et al, 2011: 986.

Synonym: Cerambyx fulvicornis Guerin, 1843.

Specimens examined: Gia Lai (Chu Se town): 1, 1.v.2011 (DIS); Dak Nong: 3 q , 10; 24.iv.2011 (DIS); Dak Lak (Pong Drang, Krong Buk): 2 , 27.iv.2011 (DIS).

Distribution: Vietnam (Kon Tum, Gia Lai, Dak Lak, Dak Nong); Laos.

\section{Genus Rhytidodera White, 1853}

Rhytidodera White, 1853. Cat. Col. Bit. Mus. 7: 132

Diagnosis: Antenna stout, shorter than body in both sexes, antennal segment 5-11 angulate externally. Prothorax rugose, unarmed. Elytra parallel, truncate at apex. Procoxa cavity weakly angulate externally. Prosternal intercoxal process sloping gradually posterior.
Hind tarsal segment 1 shorter than $2+3$ combine.

39. Rhytidodera bowringii White, 1853

Rhytidodera bowringii White, 1853: 133.

Hoang Vu Tru et al., 2011: 987.

Specimens examined: Son La (Muong Do, Phu Yen): 1q, 1ठ, 500-700m, 6-8.iv.2004, Nguyen Quoc Binh coll. (DIS); Cao Bang (Phia


coll. (DIS); Bac Kan (Ba Be NP): 3 q , 29.iv1.v.2014, 17.v.2014, Pham Hong Thai coll. (DIS); Vinh Phuc: 5, 2خ, 15.v.2004, 26.iv.2007, 3-24.v.2011, 16-24.6.2012; 2 ㅇ, 1 o $^{\lambda}$, Ngoc Thanh, Me Linh, 6.vii.2005, 3-4.v.2011 (DIS); Quang Tri (A Ngo, Dak Rong): 2ᄋ, 200400m, 1.vi.2006 (DIS); Gia Lai (Kon Ka Kinh NP, Dak Roong, KBang): 1ㅇ, 1ठ, 1230m, 1622.iii.2012; 1ㅇ, 1,060 m, 20.ii.2012 (DIS).

Distribution: Vietnam (Son La, Cao Bang, Bac Kan, Vinh Phuc, Quang Trị, Gia Lai); Assam; S.China.

40. Rhytidodera grandis Thomson, 1865

Rhytidodera grandis Thomson, 1865: Gressitt \& Rondon, 1970: 88.

Hoang Vu Tru et al., 2011: 987.

Specimens examined: Gia Lai (Chu Se town): 1ㅇ, 1.v.2011 (DIS); Dak Lak (Chu Yang Sin NP): $3{ }^{\lambda}$, 1.ix.2012 (DIS).

Distribution: Vietnam (Gia Lai, Dak Lak); Laos.

41. Rhytidodera integra Kolbe, 1886

Rhytidodera integra Kolbe, 1886: Gressitt \& Rondon, 1970: 88.

Ta Huy Thinh \& Hoang Vu Tru, 2005: 303.

Specimens examined: Bac Kan (Ba Be NP): 4ㅇ, 17.6.2007, 29.iv-1.v.2014, 19.v.2014 (DIS); Vinh Phuc: 1q, Tay Thien, 100 m, 21.v.2007; 3ㅇ, Ngoc Thanh, Me Linh, 7.vi.2003, 13.v.2013 (DIS); Ha Noi (Ba Vi NP): 19, 400 m, 2.vi.2001 (DIS); Nghe An (Qui Hop, Qui Chau): 19, 6.v.2003 (DIS); Quang Binh (U Bo, Truong Son, Quang Ninh): 2ð, 550 m, 9.v.2007 (DIS).

Distribution: Vietnam (Bac Kan, Vinh Phuc, Ha Noi, Nghe An, Quang Binh, Thua Thien- 
Hue, Quang Nam, Gia Lai); China; Korea; Laos; Myanmar; Thailand .

\section{Genus Trachylophus Gahan, 1888}

Trachylophus Gahan, 1888. Ann. Mag. Nat. Hist., (6) 2: 59.

Diagnosis: Eye large, separated by a narrow groove. Antennae a little longer than the body in the male, as long as body in female, segments 3 and 4 subcylindrical, segment 4 shorter than 3, segments 5-10 compressed and angulate ectoapically.Prothorax broader than long, narrow in front, not armed at the middle of side. Prosternum intercoxal process vertical or tuberculate posteriorly. Elytra elongate, subparallel, rounded at apex.

\section{Trachylophus acutulus Holzschuh, 2009}

Trachylophus acutulus Holzschuh, 2009: 267358

Holzschuh, 2009: 267-358.

Specimens examined: Vinh Phuc (Tam Dao NP): 1ð, 500m, 25-28.vii.2011 (DIS); Cao Bang (Phia Oac NR): 1ㅇ, 987m, 25.v-5.vi.2011 (DIS); Thua Thien-Hue (Bach Ma NP): 10ิ, 600m, 7.v.2003 (DIS).

Distribution: Vietnam (Vinh Phuc, Cao Bang, Thua Thien-Hue).

\section{Trachylophus sinensis Gahan, 1888}

Trachylophus sinensis Gahan, 1888: 60

Specimens examined: Cao Bang (Phia Oac NR): 3 ㅇ, 25.v-7.vi.2011, 25.v.2014 (DIS); Bac Kan (Ba Be NP): 3ㅇ, 1ð, 29.iv-1.v.2014 (DIS); Vinh Phuc (Tam Dao NP): 2ᄋ, 1.vi.1996, 511.v.2011; Quang Binh (Truong Son, Quang Ninh): 19, 550m, 11.v.2007; Quang Tri (Huong Phung, Huong Hoa): 1q, 1000m, 13.v.2007 (DIS); Thua Thien-Hue (A Roang, A Luoi): 1우, 4.v.2005; Quang Nam (Phuoc My, Phuoc Son): 1ㅇ, >1000m, 1.v.2005 (DIS); Gia Lai (Kon Ka Kinh NP): 1, 1060m, 18.ii.2012 (DIS); Kon Tum (Hieu, Kon Pong): 19, 800-1000m, 25.v13.vi.2006 (DIS).

Distribution: Vietnam (Cao Bang, Bac Kan, Vinh Phuc, Quang Binh, Quang Tri, Thua Thien-Hue, Quang Nam, Gia Lai, Kon Tum); China.

\section{Genus Trirachys Hope, 1841}

Trirachys Hope, 1841. Proc. Ent. Soc. Lond. 1841: 61 .

Diagnosis: Head with a partial ridge between eyes. Antenna segments 5-10 spined endoapically. Procoxa cavity weakly angulate externally. Elytral pubescence generally varied, producing highly changeable patterns according to incidence of light.

\section{Trirachys orientalis Hope, 1841}

Trirachys orientalis Hope, 1841: Gressitt \& Rondon, 1970: 68.

Ta Huy Thinh \& Hoang Vu Tru, 2005: 303.

Synonym: Trirachys formosana Schwarzer, 1925.

Specimens examined: Quang Nam (Cha Val, Nam Giang): 1ㅇ, 1ð̂, 27.iv.2005 (DIS).

Distribution: C. Vietnam (Quang Nam); S. China (Taiwan); Japan (Kyushu); Laos.

\section{Genus Xoanodera Pascoe, 1857}

Xoanodera Pascoe, 1857. Trans. Ent. Soc. Lond. ser 2,4: 92.

Diagnosis: Head triangular. Antennae stout, slightly longer than body in male, shorter in female; antennal segment 5-10 expanded externally and sharply angulate ectoapically. Prothorax as wide as head. Prosternal intercoxal process vertical. Procoxa cavity weakly angulate externally, closed posteriorly. Elytra parallel, truncate at apex.

45. Xoanodera (Lajoyeia) vitticollis Gahan, 1891

Xoanodera vitticollis Gahan, 1891: 32.

Gressitt \& Rondon, 1970: 91.

Synonym: Lajoyeiainterrupta Pic, 1933.

Specimens examined: Vinh Phuc: 29 , Tam Dao NP, 25.v.2004 (DIS); Quang Binh: 10̄, Truong Son, Quang Ninh, 500 m, 9.v.2007 (DIS); Quang Nam: 1\%, Cha Val, Nam Giang, 28.iv.2005 (DIS); Dong Nai: 6ㅇ, 6今, Vinh Cuu NR, 27-31.xii.2007 (DIS).

Distribution: Vietnam (Vinh Phuc, Quang Binh, Quang Nam, Kon Tum, Dong Nai); Laos, Borneo. 
46. Xoanodera interrupta (Pic, 1933)

Lajoyeia interrupta Pic, 1933: 10.

Heffern, 2005: 17.

Specimens examined: None.

Distribution: Vietnam, Borneo, Sumatra.

47. Xoanodera maculata Schwarzer, 1925

Xoanodera maculata Schwarzer, 1925: 21.

Hoang Vu Tru et al., 2011: 987.

Specimens examined: Son La (Co Ma, Thuan Chau): 19, 4.vi.2008 (DIS); Cao Bang (Phia Oac NR): 2q, 11.v.2010, 25.v.2014 (DIS); Phu Tho (Xuan Son NP): 19, 21.x.2004 (DIS); Vinh Phuc: 2q, Tay Thien, Tam Dao, 12.v.2004, 25.iv.2007; 2ㅇ, 20, Ngoc Thanh, Me Linh, 13.v.2013; 6ㅇ, 1§̂, Tam Dao NP, 2528.vii.2011，2.v.2013， 16-24.vi.2014 (DIS); Thanh Hoa (Lung Cao, Ba Thuoc): 1 , $700 \mathrm{~m}$, 11-13.iv.2002 (DIS); Quang Binh (Truong Son, Quang Ninh): 1, 1ふึ, 550 m, 9.v.2007 (DIS); Thua Thien-Hue (A Roang, A Luoi): 29, 700 m, 3.v.2006, 28.v.2006 (DIS); Quang Nam (Phuoc My, Phuoc Son): 1ð̂, 1.v.2005 (DIS)); Gia Lai (Kon Ka Kinh NP): 1q, 1,060 m, 18.ii.2012 (DIS).

Distribution: Vietnam (Son La, Cao Bang, Phu Tho, Vinh Phuc, Thanh Hoa, Quang Binh, Gia Lai); China.

\section{Xoanodera regularis Gahan, 1890}

Xoanodera regularis Gahan, 1890: 52-53

Ta Huy Thinh \& Hoang Vu Tru, 2005: 303.

Specimens examined: Bac Kan (Ba Be NP): 3q, 29.iv-1.v.2014 (DIS); Vinh Phuc (Ngoc Thanh, Me Linh): 29, 13.v.2013 (DIS); Ninh Binh (Cuc Phuong NP): 29, 30.iv-1.v.2006 (DIS); Quang Tri: 1q, 950m, 12.v.2007; 1, Khe Sanh, Huong Hoa, 400m, 3.vi.2006 (DIS); Thua Thien-Hue (A Roang, A Luoi): 2 , 700m, 3.v.2005 (DIS); Quang Nam (Cha Val, Nam Giang): 19, 27.iv.2005 (DIS).

Distribution: Vietnam (Bac Kan, Vinh Phuc, Ninh Binh, Quang Tri, Thua Thien-Hue, Quang Nam, Kon Tum), NE. India; Myanmar; Laos.

49. Xoanodera striata Gressitt \& Rondon, 1970

Xoanodera striata Gressitt \& Rondon, 1970: 91.
Specimens examined: Quang Tri (Huong Phung, Huong Hoa): 2今, 950 m, 12.v.2007 (DIS); Thua Thien-Hue (Bach Ma NP): $1 \hat{\jmath}$, 1200, 22.iv.2009; 5ㅇ, >1,000 m, 5-8.v.2003; Lam Dong (Lac Duong): 1q, 6.iv.2013 (DIS).

Distribution: Vietnam (Quang Tri, Thua Thien-Hue, Lam Dong); Laos.

\section{Genus Zatrephus Pascoe, 1857}

Zatrephus Pascoe, 1857. Trans. Ent. Soc. Lond. ser 2,4: 94.

Diagnosis: Head short, vertical in front. Antennae stout, as long as body in male, shorter in female; antenna segment 6 - 10 expanded externally and angulate ectoapically. Prosternum intercoxal process tuberculate and vertical at posteriorly. Elytra elongated, convex, truncate at apex.

50. Zatrephus longicornis Pic, 1930

Zatrephus longicornis Pic, 1930: 15.

Gressitt \& Rondon, 1970: 88.

Specimens examined: Vinh Phuc: $2 \hat{\jmath}$, Tam Dao NP, 500m, 25.v.2004; Thua Thien-Hue: 1§̂, Bach Ma NP, >1000m, 5-8.v.2003 (DIS).

Distribution: Vietnam (Vinh Phuc, Thua Thien-Hue); China; Laos.

\section{DISCUSSION}

Based on the analysis of 515 the longhorn beetle specimens of the tribe Cerambycini, there were 5 species newly recorded for the longhorn beetle fauna of Vietnam. Five larger genera occurred in Vietnam are Aeolesthes (14\%), Dymasius (14\%), Xoanodera (10\%), Derolus $(8 \%)$ and Neocerambyx (8\%). The species Neocerambyx vitalisi Pic, 1923 has a largest body size, specimen collected from Quang Tri province with $74 \mathrm{~mm}$ in length. In this paper some plants are reported as infested with longhorn beetle species, namely Aeolesthes holosericea (Fabricius, 1787) infested on beadtree [49]; Nadezhdiella cantori (Hope, 1845) damaged Thanh Tra pomelo in Thua ThienHue province [35].

All species of the tribe Cerambycini from Vietnam are widely distributed over the Oriental region. Among them, there are three 
species known only from Vietnam, namely Dymasius cos Holzschuh, 1998; Margites aggregatus Holzschuh, 1999 and Trachylophus acutulus Holzschuh, 2009.

There are 8 species previousely recorded from Vietnam (Gressitt \& Rondon, 1970; Heffern, 2005; Holzschuh, 1998; Holzschuh, 1999; Holzschuh, 2005; Hua Li-zhong, 2002; Pic, 1923; Pic, 1937), unfortunately, we could not find any specimens of these species.

Acknowledgements: We thank Bsc. Hoang Vu Tru, Assoc. Prof. Dr. Truong Xuan Lam (IEBR) and Dr. Pham Hong Thai, Dr. Nguyen Quoc Binh (Vietnam National Museum of Nature) for assistance with field work and providing specimens and data.

\section{REFERENCES}

1. Abang F., 2013. Cerambycidae in Sarawak. http://www.arbec.com.my/cerambycinae/. Acessessed 8.5.2014

2. Bouchard P., Bousquet Y., Davies A. E., Alonso-Zarazaga M. A., Lawrence J. F., Lyal C. H. C., Newton A. F., Reid C. A. M., Schmitt M., Slipinski S. A., \& Smith A. B. T., 2011. Family-group names in Coleoptera (Insecta). Zookeys, 88: 1-972.

3. Bousquet Y., Daniel J. H., Bouchard P., \& E. H. Nearns, 2009. Catalogue of familygroup names Cerambycidae (Coleoptera). Zootaxa, 2321: 1-80.

4. Cao Thi Quynh Nga, Khuat Dang Long, 2014. A preliminary list of the subfamily Cerambycinae (Coleoptera: Cerambycinae) of Vietnam. Tap chi Sinh hoc, 36(1): 12-38.

5. Fabricius J. C., 1775 . Systema Entomologiae, sistens insectorum classes, ordines, genera, species, adiectis synonymis, locis, descriptionibus, observationibus. Officina Libraria Kortii, Flensburgi et Lipsiae. 832 pp.

6. Gahan C. J., 1890. Descriptions of new species of Longicornia from India and Sri Lanka. The Annals and Magazine of Natural History, London, (6) 5(25): 48-66. 1 pl. 8 fig.
7. Gahan C. J., 1891. Notes on longicorn Coleoptera of the group Cerambycinae with description of new genera and species. The Annals and Magazine of Natural History, London, (6) 7(37): 19-34.

8. Gahan C. J., 1894. A list of the longicorn Coleoptera collected by Signor Fea in Myanmar and the adjoining regions with descriptions of the new genera and species. Viaggio di Leonardo Fea in Birmania e regioni vicine. Annali del Museo Civico di Storia Naturale di Genova, (2a) 14(44): 5-104.

9. Gahan C. J., 1906. The Fauna of British India including Sri Lanka and Myanmar. Coleoptera. Vol. I (Cerambycidae). London. $329 \mathrm{pp}$.

10. Gressitt J. L., 1939. A collection of longicorn beetles from T'ien-mu Shan, East China (Coleoptera: Cerambycidae). Notes sur l'Entomologie Chinoise, 6(4): 81-133.

11. Gressitt J. L., 1940. The longicorn beetles of Hainan Island. Philippine Journal of Science. Manila, 72(1/2): 1-239.

12. Gressitt J. L., 1951. Longicorn beetles of China. Longicornia vol. II. Paul Lechevalier, Paris. 677pp.

13. Gressitt J. L., Rondon J. A., 1970. Cerambycid-beetles of Laos. Pacific Insects Monograph. Bishop Museum, Honolulu, 24: 1-314.

14. Hayashi M., 1992. Study of Asian Cerambycidae, X (Coleopera). The Entomological Review of Japan, Vol XLVII, No. 2: 20p.

15. Heffern D. J., 2005. Catalog and Bibliography of Longhorned Beetles from Borneo (Coleoptera: Cerambycidae). http://www.zin.ru/animalia/Coleoptera/pdf/ borneo_catalog_electronic_version_20051.pdf. 102pp.

16. Heffern D. J., Ozdikmen H., 2007.Nomenclatural change for Oriental longhorn beetles (Coleoptera; Cerambycidae). Munis Entomology and Zoology, 2(1): 59-62.

17. Heffern D. J., 2011. Catalog and 
Cao Thi Quynh Nga, Khuat Dang Long, Ta Huy Thinh

Bibliography of Longhorned Beetles from Borneo (Coleoptera, Cerambycidae). Electronic Version, 1: 106.

18. Hoang Vu Tru, Ta Huy Thinh, 2006. Spreading of the Longhorn (Cerambycidae, Coleoptera) in the Middle part of Cetral Vietnam. The scientific conference on forward to agronomy management technology for development of sustainable agriculture in Vietnam, Hanoi, 543-550.

19. Hoang Vu Tru, Ta Huy Thinh, Cao Thi Quynh Nga, 2009. Distribution of the Longhorn beetles (Cerambycidae, Coleoptera) in the Northern Truong Son range passing through Thanh Hoa, nghe An and $\mathrm{Ha}$ Tinh provinces. Proceeding of the $3^{\text {rd }}$ national scientific conference on Ecology and Biological Resources. Agriculture Publishing House, Hanoi: 394-400.

20. Hoang Vu Tru, Ta Huy Thinh, Cao Thi Quynh Nga, 2011. Result of the survey on longhorn beetles (Cerambycidae, Coleoptera) along the HoChiMinh road through Tay Nguyen highland. Proceeding of the $4^{\text {nd }}$ national scientific conference on Ecology and Biological Resources, Agriculture Publishing House, Hanoi (ISSN 1859-4425). 982-988.

21. Holzschuh C., 1989. Beschreibung von 20 neuen Bockkafern aus Thailand, Vietnam und Taiwan (Coleoptera, Cerambycidae). Entomologica Basiliensia, 13: 361-390.

22. Holzschuh C., 1991. Neue Bockkäfer aus Asien II. 63 neue Bockkäfer aus Asien, vorwiegend aus China und Thailand (Coleoptera: Disteniidae und Cerambycidae). FBVA Berichte, Schriftenreihe der Forstlichen Bundesversuchsanstalt. Vol. 60. Wien, Austria. 80 pp., 80 ill.

23. Holzschuh C., 1992. 57 neue Bockkafer aus Asien, vorwiegend aus China, Thailand und Vietnam (Coleoptera, Cerambycidae). Forstlicen Bundesversuchsanstalt. 63p.

24. Holzschuh C., 1995. Description of 65 new longhorned beetles from europe and asia, mostly from Thailand and China
(Coleoptera: Disteniidae and Cerambycidae). FBVA - Berichte, 84: 64p.

25. Holzschuh C., 1998. Description of 68 new longhorned beetles from asia, mostly from Thailand and China (Coleoptera: Disteniidae and Cerambycidae). FBVABerichte, 107: 65p.

26. Holzschuh C., 1999. Description of 71 new longhorned beetles from Asia, mostly from China, Laos, Thailand and India (Coleoptera: Cerambycidae). FBVABerichte, 110: 64p.

27. Holzschuh C., 2003. Description of 72 new longhorned beetles from Asia, mostly from China, Laos, Thailand and India (Coleoptera: Cerambycidae). Entomologica Basiliensia, 25: 147-241.

28. Holzschuh C., 2005. Description of new longhorned beetles from SE Asia and Borneo (Coleoptera: Cerambycidae). Les Cahiers Magellanes, 46: 41p

29. Holzschuh C., 2007. Description of 80 new longhorned beetles from Orientalis and Paleartic, mostly from China, Laos and Borneo (Coleoptera: Cerambycidae). Entomologica Basiliensia et Collectionis Frey, 29: 177-286.

30. Holzschuh C., 2009. Description of 59 new longhorned beetles from Gattungen, Orientalis and Paleartic, mostly from China, Laos and Borneo (Coleoptera: Cerambycidae). Entomologica Basiliensia et Collectionis Frey, 31: 267-358.

31. Holzschuh C., 2010. Description of 66 new longhorned beetles from Gattungen and Orientalis, mostly from China, Laos and Thailand (Coleoptera: Cerambycidae). Entomologica Basiliensia et Collectionis Frey, 32: 137-225.

32. Hoppe P., Lameere M., 1919. Traite D'Entomologie Indochinoise. Cerambycidae. Impr. Minsang, Hanoi: 141-148.

33. Hua Li-zhong, 2002. List of Chinese Insects. Vol. II. Family Cerambycidae. Zhongshan (Sun Yat-sen) University Press: 189-237. 
34. Hua Li-zhong, Hajime N., Saemulson G. A., \& Lingafelter S. W., 2009. Iconography of Chinese Longicorn Beetles (1406 species) in Color. Series Publication of Museum of Biology. Sun Yat-sen University Press. 475p.

35. Le Trong Son, 2007. The species composition and biological characteristics of mainly pests Thanh Tra pomelo from Thua Thien-Hue. Problems of basic research in life sciences in Qui Nhon city. Science and technics publishing house: 158-161.

36. Linnaeus C., 1758. Systema naturae per regna tria naturae secundum classes, ordines, genera, species cum characteribus, differentiis, synonymis, locis. Tomus I. Editio Decima, reformata. Laurentii Salvii, Holmiae, 1(10): 824 pp.

37. Makihara H., Mannakkara A., Fujimura T., and Ohtake A., 2008. Checklist of longicorn coleopterea of Sri Lanka (1) Vesperidae and Cerambycidae including Lamiinae. Bulletin of FFPRI. Vol. 7 No. 2 (No. 407): 95-110.

38. Mai Phu Qui, Tran Thi Lai, Tran Tran Bich Lan, 1981. The survey of animals in North Vietnam. Family Longhorned-beetles (Cerambycidae, Coleoptera). Science and TechnologyPublishing House, Hanoi: 131137.

39. Newman E., 1842. Cerambycitum insularum manillarum Dom. Cuming captorum enumeratio digesta. The Entomologist. London, 1(15): 243-248.

40. Pascoe F. P., 1856. Descriptions of new genera and species of Asiatic longicorn Coleoptera. The Transactions of the Entomological Society of London, 4(2): 4250. 1 pl. (XVI).

41. Pascoe F. P., 1857. On new genera and species of longicorn Coleoptera. Part II. The Transactions of the Entomological Society of London, 4(2): 89-112. 2 pls. (XXII-XXIII).

42. Pascoe F. P., 1858. On new genera and species of longicorn Coleoptera. Part III. The Transactions of the Entomological Society of London, 4(2): 236-266. 2 pls. (XXV-XXVI).
43. Pascoe F. P., 1859. On new genera and species of Longicorn Coleoptera. Part IV. The Transactions of the Entomological Society of London, 5(2): 12-61. 1 pl. (II).

44. Pascoe F. P., 1864. Longicornia Malayana; or, a Descriptive Catalogue of the Species of the three Longicorn Families Lamiidae, Cerambycidae and Prionidae collected by Mr. A. R. Wallace in the Malay Archipelago. (Part I). The Transactions of the Entomological Society of London, 1(3): 1-96.

45. Pascoe F. P., 1865. Longicornia Malayana; or, a Descriptive Catalogue of the Species of the three Longicorn Families Lamiidae, Cerambycidae and Prionidae collected by Mr. A. R. Wallace in the Malay Archipelago. (Part II). The Transactions of the Entomological Society of London, 2(3): 97-224.

46. Pascoe F. P., 1866. Longicornia Malayana; or, a Descriptive Catalogue of the Species of the three Longicorn Families Lamiidae, Cerambycidae and Prionidae collected by Mr. A. R. Wallace in the Malay Archipelago. (Part III). The Transactions of the Entomological Society of London, 3(3): 225-336.

47. Pascoe F. P., 1867. Longicornia Malayana; or, a Descriptive Catalogue of the Species of the three Longicorn Families Lamiidae, Cerambycidae and Prionidae collected by Mr. A. R. Wallace in the Malay Archipelago. (Part IV). The Transactions of the Entomological Society of London, 3(3): 337-464.

48. Pascoe F. P., 1868. Longicornia Malayana; or, a Descriptive Catalogue of the Species of the three Longicorn Families Lamiidae, Cerambycidae and Prionidae collected by Mr. A. R. Wallace in the Malay Archipelago. (Part V). The Transactions of the Entomological Society of London, 3(3): 465-498.

49. Pham Quang Thu, Le Van Binh, 2010. Theblack-silver longhorn beetles Aeolesthes holosericea (Fabricius, 1787) harmed bead- 
tree. Journal of agricultural and rural development, 8: 96-101.

50. Pic M., 1923. Nouveautés diverses. Mélanges Exotico-Entomologiques. Moulins, 38:1-32.

51. Pic M., 1933. Nouveautés diverses. Mélanges Exotico-Entomologiques. Moulins, 62:1-36.

52. Plant Protection Research Institute, 1976. Result of the survey of insect (1967-1968), Family Cerambycidae. Agriculture Publishing House, Hanoi: 213-229.

53. Schwarzer B., 1925. Sauters FormosaAusbeute (Cerambycidae. Col.). (Subfamilie Cerambycinae). Entomologische Blätter. Zeitschrift für Biologie und Systematik der Käfer. Berlin \& Krefeld, 21: 20-30.

54. Ta H. T., Hoang V. T., 2005. List of longhorn collected on Truongson moutains from Quangtri, Thua thien - Hue and Quangnam province. Proceedings, The $2005^{\text {th }}$ National Conference on Life Sciences. Science and Techniques Publishing House, Hanoi, 302304.

55. Thomson J., 1860. Essai d'une classification de la famille des cérambycides et matériaux pour servir à une monographie de cette famille. Paris. 396 pp.

56. Thomson J., 1861. Essai d'une classification de la famille des cérambycides et matériaux pour servir à une monographie de cette famille. Paris. 396 pp.

57. Thomson J., 1864. Systema cerambycidarum ou exposé de tous les genres compris dans la famille des cérambycides et familles limitrophes. H. Dessain, Liège, 578pp.

58. Vitali F., 2007. About the taxonomic status of some species of the genus Aeolesthes Gahan, 1890 (Coleoptera, Cerambycidae). Entomapeiron (N. S.), 1(3): 65-80.

59. Vitalis de Salvaza R., 1919. Traité d'entomologie indochinoise. Cerambycidae. Impr. Minsang, Hanoi: 139-162.

60. White A., 1853. Catalogue of coleopterous insects in the collections of the British museum, pt VII. Longicornia I. pp. 1-174. pl. 1-4. London.

61. http://farangsgonewild.weebly.com/chec klist-cerambycidae-long-horned-beetlesfrom-thailand.html

\title{
DANH SÁCH LOÀI VÀ NHŨ̉NG GHI NHẬN MỚI TRONG TỌC XÉN TÓC Cerambycini (Coleoptera: Cerambycidae: Cerambycinae) CỦA VIẸT NAM
}

\author{
Cao Thị Quỳnh Nga, Khuất Đăng Long, Tạ Huy Thịnh \\ Viện Sinh thái và Tài nguyên sinh vật, Viện Hàn lâm KH\&CN Việt Nam
}

\section{TóM TẮT}

Dựa trên phân tích 515 mẫu xén tóc hiện có tại Phòng Hệ thống học côn trùng, Viện Sinh thái và Tài nguyên sinh vật, bài báo này đưa ra danh sách 50 loài Xén tóc của 18 giống thuộc tộc Cerambycini ở Việt Nam. Trong số đó, đã ghi nhận mới 5 loài cho khu hệ Xén tóc của Việt Nam, đó là Aeolesthes (Pseudaeolesthes) pericalles Gressitt \& Rondon, 1970; Aeolesthes sinensis Gahan, 1890; Derolus ornatus Gressitt \& Rondon, 1970; Dymasius maculatus Gressitt \& Rondon, 1970 và Massicus raddei (Blessig, 1872). Bài báo còn đưa ra khóa định loại bằng ảnh chụp minh họa 18 giống của tộc Cerambycini. Đặc điểm chẩn loại giống, thông tin về vật mẫu và phân bố của loài cũng được trình bày trong bài báo này.

Tù khóa: Danh sách loài, ghi nhận mới, khu hệ, phân bố, xén tóc.

Ngày nhận bài: 10-7-2014 